\title{
X-ray tomography of the aerospace products
}

\author{
Vladimir Smolyanskiy ${ }^{1, *}$, Maxim Rychkov ${ }^{1}$, and Valeriy Borikov ${ }^{1}$ \\ ${ }^{1}$ National Research Tomsk Polytechnic University, 634050 Tomsk, Russia
}

\begin{abstract}
Currently, non-destructive methods are increasingly being used to inspect the critical components in different branches of industry. Special focus is on the usage of high-energy X-ray microtomography used to inspect large objects with high density. This method provides high accuracy and a possibility to measure the internal and external dimensions of the test sample without its destruction. It allows obtaining the information about internal defects and damages as well as manufacturing and assembling quality. This article describes a tomographic study of a medium-size high-density sample with a resolution about of 70 microns.
\end{abstract}

\section{Introduction}

The quality control of aerospace products is one of the steps in their manufacturing process. During this phase, operational safety and product reliability are evaluated.

Despite the variety of known methods [1-2], the study of high-density objects remains an unsolved problem, because product thickness and dimensions prevent from carrying out an inspection without sample destruction. A solution came with the discovery of X-ray testing and computed tomography (CT) [3].

The main objective is to detect defects in the internal ferrite rings of a rotor position sensor with a diameter of $3 \mathrm{~mm}$ and to measure the depth of a 1 [mm] weld. Reliability and failure-free service life of the electro motor depend on manufacturing and assemble quality control. It requires inspection of the motor assembly, which is only possible with X-ray tomography.

\section{Materials and methods}

The object of the study is the motor DBE63 designed for operation in electric pump (LZTK-2) in the thermal control system of a spacecraft with on-orbit operating lifetime of about 15 years. The motor is $90 \mathrm{~mm}$ in diameter and consists of titanium and aluminium parts with a thickness ranging from 6 to $12 \mathrm{~mm}$ (Figure. 1).

The inspection is performed on a cone-beam computed tomography scanner (CBCTscanner) for large objects designed by Tomsk Polytechnic University (TPU). The CBCTscanner consists of an X-ray source MXR-451HP/11 (COMET), a flat panel detector XRD1642 (PerkinElmer) and a linear rotary table DCNI 50 ST (DELKEN).

*Corresponding author: vsmol@tpu.ru 

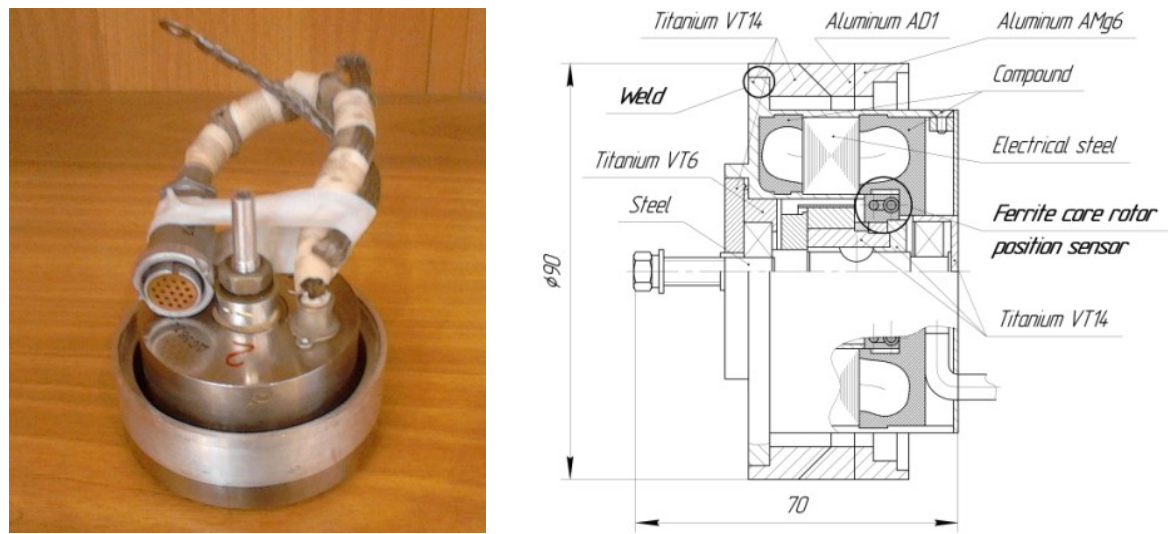

Fig. 1. Electro motor DBE63.

The cone-beam method provides high rendering performance and allows varying the sample dimensions and spatial resolution [3] (Figure. 2).

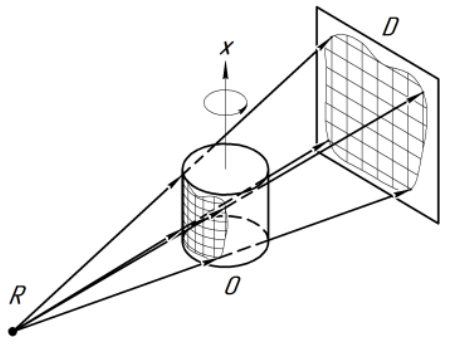

Fig. 2. Schematic diagram of the cone-beam method Legend: $\mathrm{R}$ - X-ray source, $\mathrm{O}$ - test sample, $\mathrm{D}$ detector, $\mathrm{x}$ - rotation axis.

According to the thickness of the inspected material, the scan mode is set to: $300 \mathrm{kV}$ accelerating voltage, $2.3 \mathrm{~mA}$ anode current, $0.3 \mathrm{deg}$ projection angle increment, $1 \mathrm{~s}$ exposure period, 720 projections, 45 min data collection time.

The INKCT software package (TPU) collects shadow projections. The NRecon software package (Skyscan) performs the 3D reconstruction (Figure. 3.a) by filtered back projection [5, 6]. Finally, the CTvox software package (Skyscan) implements further volumetric visualization of the reconstructed slices (Figure.3.b).
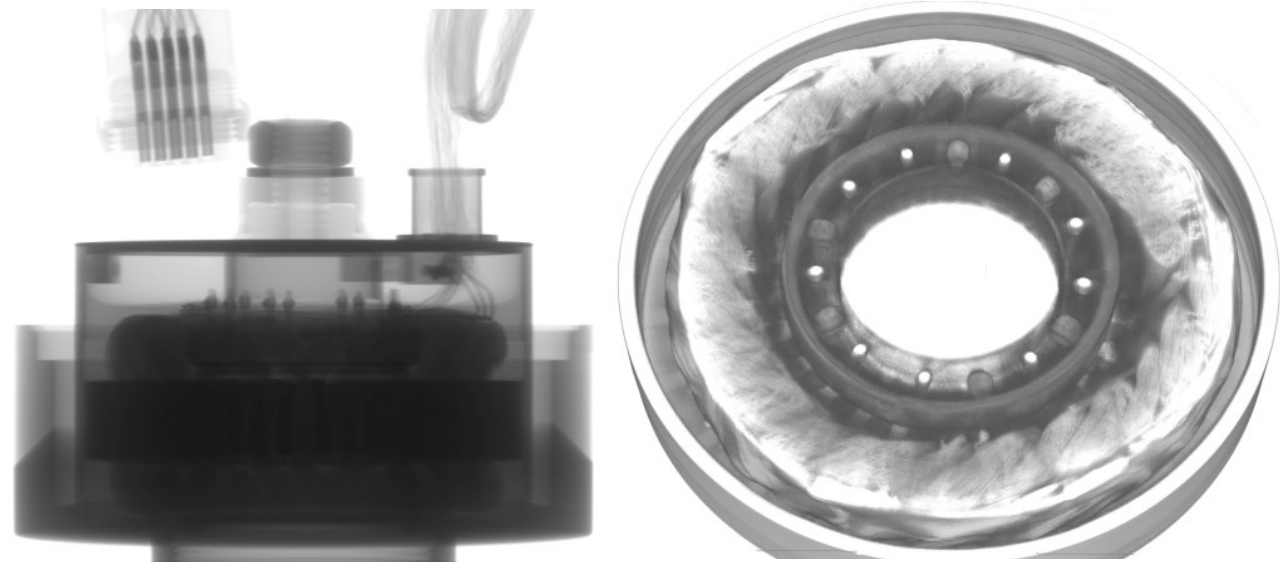

Fig. 3. a) Motor shadow projection, b) Motor cross-section near the ferrite rings. 


\section{Results}

Computed tomography, reconstruction, segmentation and visualisation are performed to study the object in detail. In addition to the outer surface of the sample, the model shows its internal elements in accordance with their density. Cross-section images of the complex internal structure bring hidden defects into clearer focus without any destructions of the electro motor. The analysis results show the position of ferrite rings, their dimensions (diameter of $2.986 \mathrm{~mm}$ ) and clear boundaries. The inspection reveals the cause of the trouble in motor operation. There is one of the ferrite rings with a through-thickness crack (Figure. 4).
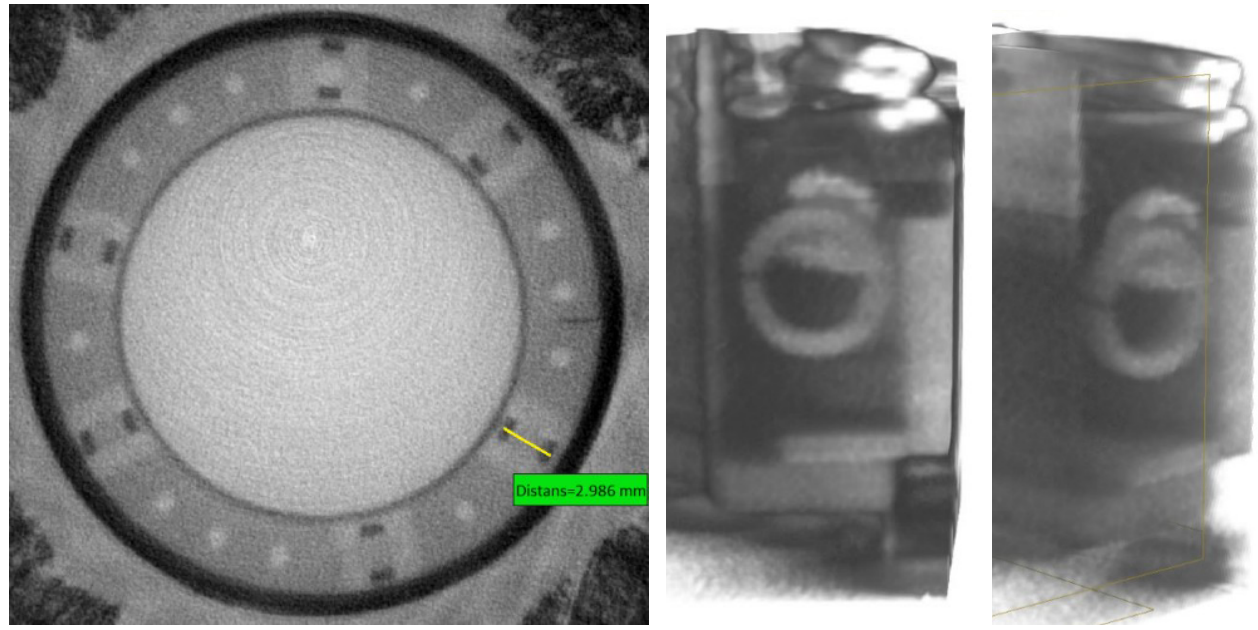

Fig. 4. Through-thickness cracked ferrite ring.

The internal structure of the object was reconstructed to inspect the weld of the motor cover. The images were obtained at the X-ray tube voltage $225,250,300,350 \mathrm{kV}$ and current 3.0, 2.5, 2.3, $2.0 \mathrm{~mA}$, respectively. We did not find the weld on the reconstructed slice (Figure 5) by comparing the detail drawing and the object shadow projection.

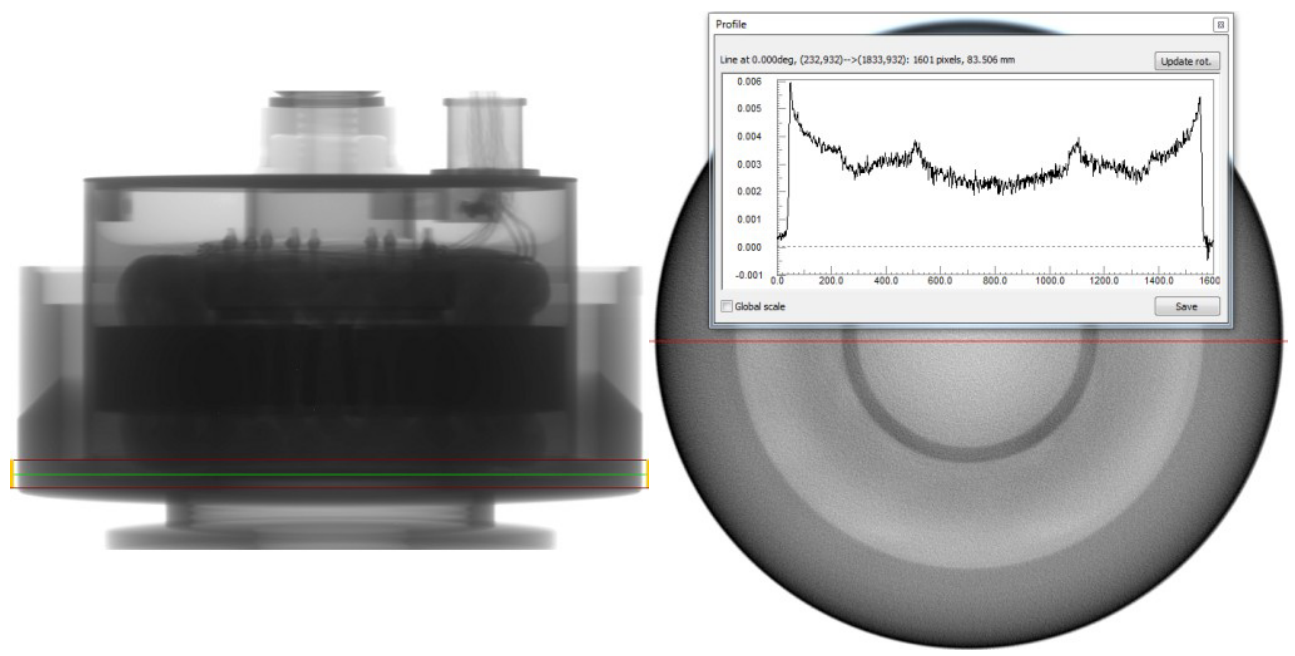

Fig. 5. The object shadow projection and the reconstructed slice with the weld. 


\section{Discussion}

For the given voltage and current, the weld is not defined. This is caused by the low penetrating power of radiation and the lack of differences in density (weld placed between titanium alloys VT14).

Since the base material of the construction is titanium (a high-density material) and the size of the weld is $1 \mathrm{~mm}$, the CBCT-scanner must use a more powerful ionizing radiation source with harder rays and higher penetrating power, for example, with a betatron MIB-4 (TPU) and linear array detector IHE2 (Detection Technology). It is necessary to provide an $\mathrm{X}$-ray beam hardening by special copper filters that absorb soft radiation. It would increase the average power and improve the reconstruction quality. An X-ray beam must be collimated by lead blocks to reduce the scattered beam influence and to provide a high spatial resolution. The scan settings for a new installation should be approximately as follows: accelerating voltage $-500 \mathrm{kV}$, projection angle increment $-0.3 \mathrm{deg}$, exposure period $-3-5 \mathrm{~s}$.

\section{Conclusions}

$\mathrm{CT}$ is a powerful tool to study the internal structure of an object. In this article, we describe the tomographic scanning of the space-worthy electro motor DBE63 as well as its following reconstruction and analysis. This experiment allows us to inspect a medium-size highdensity sample with a resolution of about 70 microns and to detect product defects affecting its performance.

However, further study will focus on the possibility of using high-energy highresolution X-ray microtomography for the inspection of a large-size high-density object. A promising new application for the future is using of a betatron will be as a more powerful ionizing radiation source with harder rays and higher penetrating power. This work shows the importance of X-ray tomography for non-destructive testing in the space industry. The results of this work demonstrate the efficiency of the developed CT system.

\section{References}

1. V. P. Vavilov, D. D. Burle, NDT\&E Int. 73, 28 (2015)

2. V. N. Borikov, O. V. Galtseva, G. A. Filippov, J. Phys.: Conf. Ser. 671, 012014 (2016)

3. S. V. Chakhlov, S. P. Osipov, A. K. Temnik, V. A. Udod, Rus. J. of Nondestructive Testing 52, 235 (2016)

4. T.M. Buzug, Computed Tomography: From Photon Statistics to Modern Cone-beam CT (Springer Science amp; Business Media, Berlin, 2008)

5. B. Wolkowski, E. Snead, M. Wesolowski, J. Singh, M. Pettitt, R. Chibbar, S. Melli and J. Montgomery, J. of Synchrotron Radiation 22, 1130 (2015)

6. Feldkamp L. A., Davis L. C., Kress J. W., JOSA A. 1, 612 (1984) 